\title{
Evaluación y valoración del Estado Nutricional en estudiantes adolescentes de Ceuta. Estudio ABYLA
}

Adrián Ruiz Laso. Grupo de investigación HUM-840: "Desarrollo infantil y adolescente. Factores de riesgo". Campus Universitario de Ceuta, Universidad de Granada

Recepción: 8 de noviembre de 2015 | Revisión: 9 de noviembre de 2015 | Aceptación/Publicación: 10 de noviembre de 2015 Correspondencia: adrianruizlaso@yahoo.es

Citar: Ruiz-Laso, A. (2015). Evaluación y valores del Estado Nutricional en estudiantes adolescentes de Ceuta. Estudio ABYLA. ReiDoCrea, 4, 370-377. [http://hdl.handle.net/10481/38768]

Resumen: La malnutrición en la adolescencia es un importante determinante de salud presente y futura del individuo. Estudios de ámbito nacional como ENKID y la Estrategia NAOS no han incluido a la población adolescente de Ceuta. Otros como la Encuesta Nacional de Salud trabajan con medidas antropométricas auto-referidas. Objetivos: conocer el estado nutricional y la prevalencia de malnutrición en los adolescentes ceutíes con edades de 13 a 19 años. Trasladar estos conocimientos a la planificación de posibles intervenciones en los ámbitos de la Promoción y de la Educación para la salud. Método: Estudio epidemiológico descriptivo, transversal sobre una muestra representativa de la población adolescente ceutí de 507 jóvenes, mujeres y varones de culturas cristiana y musulmana. Se midieron el peso, la altura, los perímetros de cintura, cadera, muñeca y mesobraquial, y se halló la composición corporal. Resultados: la prevalencia de infrapeso es del $10,6 \%$, el sobrepeso alcanza un $23,62 \%$, y la obesidad el $10 \%$. Por sexo y cultura, la prevalencia de infrapeso es mayor en varones que en mujeres, y en musulmanes que en cristianos. La obesidad es más alta en mujeres que en varones, y en musulmanes que en cristianos. Conclusiones: de cada diez jóvenes uno presenta infrapeso, dos sobrepesos y uno obesidad. Siendo la prevalencia de sobrepeso y de infrapeso superior a la media nacional, y la prevalencia de obesidad inferior. Estas referencias facilitarán la implementación de programas de Educación para la salud.

Palabras clave: Malnutrición | Educación

Evaluation and Assessment of Nutritional Status in Adolescent Students of Ceuta. Study ABYLA

Abstract: Malnutrition during adolescence is paramount for present and future health of the individual. National studies such as ENKID or the NAOS Strategy have not included the teenage population of Ceuta. Others like the National Health Survey are based on self-reported anthropometric measures. Objectives: to determine the nutritional status and the prevalence of malnutrition in Ceuta adolescents aged 13-19 years. To transfer this knowledge to the planning of possible interventions in the areas of Promotion and Education for Health. Method: Descriptive, transversal epidemiological study on a representative sample of the population of 597 young teenagers from Ceuta, women and men of Christian and Muslim cultures. Weight, height, and circumference of the waist, hip, wrist and mesobranchial were measured, and body composition was assessed. Results: The prevalence of underweight is $10,6 \%$, overweight reaches $23,62 \%$, and obesity $10 \%$. By sex and culture, the prevalence of underweight is higher in men than in women and in Muslims than in Christians. Obesity is higher in women than in men and in Muslims than Christians. Conclusions: Out of ten teenagers, one is underweight two overweight and one shows obesity, being the prevalence of overweight and underweight higher than the national average, and lower the one of obesity. These references will facilitate the implementation of programs of education for health.

Keywords: Malnutrition | Education

\section{Introducción}

Los estudios sobre las malnutriciones y los hábitos cotidianos de los adolescentes que determinan su salud no son abundantes porque históricamente no se ha considerado a la adolescencia como una etapa vulnerable, frente a la gran importancia concedida a la adolescencia. Esta consideración ha cambiado sustancialmente hasta el punto de tratar a la adolescencia como un estadio de la persona crítico para el desarrollo de la malnutrición juvenil, y para fijar/consolidar hábitos poco saludables en el ámbito de la alimentación, e iniciarse en otros como el consumo de alcohol, tabaco y otras drogas.

A partir de la década de los 80 la preocupación por el exceso de peso se ha hecho patente tanto a nivel mundial como a niveles nacionales y regionales. En 2004 la OMS adopta la Estrategia sobre Desnutrición, Obesidad, Actividad física y Salud. Con esta decisión se insta a todas las partes interesadas para que actúen a todos los niveles sociales. Cómo traslación a España, el Gobierno pone en marcha la Estrategia NAOS ${ }^{1}$ 
(2005). En el año 2011 la ONU aprueba la Declaración Política sobre la Prevención y el Control de las Enfermedades No Transmisibles (ENT), reconociendo la importancia capital de reducir la prevalencia de malnutrición y la inactividad física.

En España, estudios de ámbito nacional como ENKID ${ }^{14}$, AVENA, DORICA y PERSEO no han incluido a los adolescentes de Ceuta ${ }^{3,4}$. Otros como HBSC y las Encuestas Nacionales de Salud no remiten datos por separado de Ceuta y de Melilla o la hacen con muestras muy pequeñas y con medidas auto-referidas, que pueden sesgar el resultado de la evaluación del estado nutricional.

Se hace necesario cubrir esta importante carencia de datos sobre la prevalencia de la delgadez, del sobrepeso y de la obesidad en los estudiantes adolescentes de Ceuta de 13 a 19 años, mediante múltiples mediciones directas y teniendo en cuenta el carácter multicultural de la población ceutí. El conocimiento sobre su estado nutricional hará posible la implementación y el desarrollo de intervenciones eficaces ámbitos educativos y de salud comunitaria.

\section{Método}

Se ha diseñado un estudio epidemiológico observacional descriptivo, transversal o de prevalencia, que se ha llevado a cabo de junio de 2013 a junio de 2014. La población de estudio ha sido la totalidad de los adolescentes escolarizados en la Ciudad Autónoma de Ceuta desde $2^{\circ}$ de la ESO hasta $2^{\mathrm{a}}$ de Bachillerato en régimen diurno. Esta población total asciende a 4052 jóvenes (Fuente MECD).

El tamaño de la muestra se calculó para valor alfa $=0,05$, con intervalo de confianza del $95 \%$, pronosticando una tasa de no respuesta del 0,06 . La representatividad y el tamaño de la muestra se hallaron con el programa estadístico de la Creative Research System, de acceso libre. Con estos criterios se fijó el tamaño de la muestra en una horquilla entre 450 y 500 adolescentes, lo que supone un porcentaje del $12,5 \%$ sobre la población total. El tamaño definitivo es de 507 adolescentes, de los que un 48,3\% son cristianos, un 49,7 musulmanes, y un $2 \%$ de otras culturas (Tabla 1 ).

\begin{tabular}{|c|c|c|c|c|c|c|}
\hline \multicolumn{7}{|c|}{$\begin{array}{c}\text { Tabla } 1 \\
\text { Distribución de la muestra por edad, sexo y cultura }\end{array}$} \\
\hline \multirow[t]{2}{*}{ Edad } & \multirow{2}{*}{$\begin{array}{c}\text { Sexo } \\
M=\text { mujer } \\
V=\text { varón }\end{array}$} & \multicolumn{3}{|c|}{ Cultura } & \multirow{2}{*}{$\begin{array}{l}\text { Total diferenciados por } \\
\text { sexo y edad }\end{array}$} & \multirow{2}{*}{$\begin{array}{c}\text { Total } \\
\text { por edad }\end{array}$} \\
\hline & & Cristiana & Musulmana & Otras & & \\
\hline \multirow[t]{2}{*}{13} & M & 20 & 21 & 0 & 41 & \multirow{2}{*}{65} \\
\hline & V & 11 & 12 & 1 & 24 & \\
\hline \multirow[b]{2}{*}{14} & $\mathrm{M}$ & 14 & 31 & 0 & 45 & \multirow[b]{2}{*}{78} \\
\hline & $\mathrm{V}$ & 17 & 16 & 0 & 33 & \\
\hline \multirow[t]{2}{*}{15} & M & 24 & 30 & 0 & 54 & \multirow[b]{2}{*}{83} \\
\hline & V & 13 & 15 & 1 & 29 & \\
\hline \multirow[t]{2}{*}{16} & $\mathrm{M}$ & 45 & 29 & 3 & 77 & \multirow[b]{2}{*}{129} \\
\hline & V & 24 & 27 & 1 & 52 & \\
\hline \multirow[t]{2}{*}{17} & M & 30 & 23 & 0 & 53 & \multirow[b]{2}{*}{98} \\
\hline & V & 25 & 17 & 3 & 45 & \\
\hline \multirow[t]{2}{*}{18} & $\mathrm{M}$ & 6 & 5 & 0 & 11 & \multirow[b]{2}{*}{21} \\
\hline & V & 5 & 5 & 0 & 10 & \\
\hline \multirow[t]{2}{*}{19} & $\mathrm{M}$ & 8 & 10 & 1 & 19 & \multirow{4}{*}{33} \\
\hline & V & 3 & 11 & 0 & 14 & \\
\hline \multirow{2}{*}{$\begin{array}{c}\text { Total por } \\
\text { cultura y sexo }\end{array}$} & $\mathrm{M}$ & 147 & 149 & 4 & & \\
\hline & V & 98 & 103 & 6 & & \\
\hline Total por cultura & & 245 & 252 & 10 & & otal muestra 5 \\
\hline
\end{tabular}


El criterio de inclusión ha sido de adolescentes escolarizados de 13 a 19 años que voluntariamente quisieran participar. Como criterio de exclusión, la negativa a participar en la investigación por no someterse a las medidas antropométricas completas. También se excluyen los diez adolescentes de otras culturas por ser una muestra poco representativa.

Se aseguró en la técnica de muestreo aleatorio simple que los alumnos adolescentes participantes estuviesen distribuidos por los diferentes sectores que conforman la Ciudad de Ceuta, seleccionando para ello a cuatro Institutos de Enseñanza Secundaria: "Abyla", "Luis de Camóens", Puertas del Campo" y "Siete Colinas". Las medidas antropométricas (Cineantropometría) se han tomado según las recomendaciones del programa Internacional de Biología (IBP), la Sociedad Española de Nutrición Comunitaria $(S E N C)^{7}$, y la ISAK (The International Society for the Advancement Kinanthropometry) practicadas en el lado derecho.

Los instrumentos de medida han sido: tallímetro modelo SECA 220, con precisión de $1 \mathrm{~mm}$, incorporado a la báscula SECA 769; tallímetro portátil 213; cinta antropométrica SECA 201; Paquímetro de acero inoxidable Rost-Frei de $1 \mathrm{~mm}$ de precisión. La composición corporal se ha estudiado con el aparato BODYSTAT 1500 de impedancia bioeléctrica tetrapolar.

Las variables analizadas han sido la edad, el sexo, la cultura, la situación laboral (padre y madre), el nivel socioeconómico, la altura, el peso, el índice de masa Corporal (IMC), los perímetros del brazo medio, de la cintura, de la muñeca, de la cadera ${ }^{15}$, la composición corporal en grasa ${ }^{9}$, la masa magra, el porcentaje de agua, el gasto energético en reposo, el requerimiento energético estimado, y el riesgo cardiovascular ${ }^{11}$. Todas estas variables fueron tomadas con la metodología de mediciones directas y estandarizadas. Los lugares para ello fueron los laboratorios de biología de los respectivos centros educativos. Necesitándose para cada adolescente un tiempo medio de 30 minutos.

Con el claro objetivo de que los datos obtenidos en la presente investigación fuesen fácilmente comparables con otros de similar temática, hemos utilizado para definir las malnutriciones los puntos de corte recomendados por diversas instituciones de prestigio en este campo, tanto a nivel mundial como a niveles nacionales y basados en curvas percentiladas ${ }^{2}$. A nivel mundial las curvas de la OMS como "referente oro", las curvas IOTF, y las curvas CDC. A nivel nacional las curvas y tablas de la Fundación Orbegozo ${ }^{8}$.

El procesamiento de la información y el análisis estadístico se ha llevado a cabo mediante las aplicaciones del programa Excel para gráficas y tablas vivariables. Los procesos de análisis estadístico, tanto descriptivo como inferencial, se ha realizado mediante el paquete software PSPP de acceso libre.

\section{Resultados}

En la población adolescente de Ceuta hallamos una prevalencia de malnutrición por infrapeso o delgadez, según el IMC y con puntos de corte OMS, del $\mathbf{9 , 4 5 \%}$. Superior en varones $(12,19 \%)$ que en mujeres $(6,72 \%)$. Por cultura y sexo, el infrapeso es más frecuente en las mujeres musulmana $(9,2 \%)$ que en las mujeres cristianas $(4,25 \%)$, y en los varones musulmanes $(13 \%)$ que en los varones cristianos $(11,38 \%)$, si bien en el caso de los varones la diferencia de prevalencia de infrapeso es menor que en las mujeres. Por edades, la edad de 13 años es la que presenta en varones mayor prevalencia de infrapeso. En las mujeres la mayor prevalencia se da en edades de 15 
y 16 años. Por sexo, cultura y nivel socioeconómico, las mujeres musulmanas con infrapeso se incluyen mayoritariamente en un nivel bajo y las cristianas en un nivel medio. Igual distribución encontramos en los varones.

La prevalencia de sobrepeso en los jóvenes adolescentes es del $\mathbf{2 3 , 6 2} \%$ con puntos de corte OMS, superior en mujeres $(26 \%)$ que en varones $(21,24 \%)$. Por sexo y cultura, las adolescentes cristianas presentan mayor prevalencia de sobrepeso $(30 \%)$ que las musulmanas (22\%). Los varones cristianos tienen una prevalencia de sobrepeso del $25,3 \%$, mayor que los varones musulmanes con un $17,18 \%$. Por cultura y nivel socioeconómico, el sobrepeso en varones musulmanes es más frecuente en el nivel medio-bajo, y en varones cristianos en el medio. En mujeres, tanto musulmanas como cristianas, la mayor frecuencia de sobrepeso se encuentra en el nivel social medio-bajo.

La prevalencia de Obesidad presenta una banda de valores entre el $7,2 \%$ y el $10 \%$, según el criterio de corte que se utilice. Por sexo y cultura, las adolescentes musulmanas alcanzan mayor prevalencia que las cristianas. Igual ocurre con los varones Por cultura y nivel socioeconómico, la prevalencia de obesidad es más frecuente en los jóvenes adolescentes de nivel medio-bajo, independientemente de su cultura (Tabla 2).

\begin{tabular}{|c|c|c|c|c|c|c|c|}
\hline \multicolumn{8}{|c|}{$\begin{array}{c}\text { Tabla } 2 \\
\text { Prevalencias nutricionales en los adolescentes de Ceuta, por sexos y criterios de puntos de corte, expresadas en } \%\end{array}$} \\
\hline & & DELGADEZ & SOBREPESO & OBESIDAD & EXCESO PESO & MALNUTRICIÓN & NORMOPESO \\
\hline \multirow[t]{3}{*}{ FO } & mujeres & 7,74 & 22,24 & 11,27 & 33,51 & 41,25 & 58,75 \\
\hline & varones & 13,45 & 15,95 & 4,58 & 20,53 & 34 & 66 \\
\hline & Media F & 10,6 & 19,1 & 7,9 & 27 & 37,6 & 62,4 \\
\hline \multirow[t]{3}{*}{ OMS } & mujeres & 6,72 & 26 & 9,44 & 35,45 & 42,18 & 57,82 \\
\hline & varones & 12,19 & 21,24 & 10,66 & 31,9 & 44 & 56 \\
\hline & Media $\mathbf{F}$ & 9,45 & 23,62 & 10 & 33,68 & 43,13 & 56,87 \\
\hline \multirow[t]{3}{*}{$\mathrm{CDC}$} & mujeres & 4,51 & 23,31 & 7,84 & 31,15 & 35,7 & 64,3 \\
\hline & varones & 10,9 & 19,85 & 10 & 30 & 40,9 & 59,1 \\
\hline & Media $\mathbf{F}$ & 7,7 & 21,58 & 8,92 & 30,57 & 38,3 & 61,7 \\
\hline \multirow[t]{3}{*}{ IOTF } & mujeres & 6,12 & 25,24 & 7 & 32,29 & 38,41 & 61,59 \\
\hline & varones & 10,27 & 22,8 & 7,49 & 30,27 & 40,6 & 59,4 \\
\hline & Media F & 8,19 & 24 & 7,26 & 31,28 & 39,5 & 60,5 \\
\hline
\end{tabular}

En síntesis, aplicando como medida el IMC percentilado, la suma de las malnutriciones alcanza unos valores medios de prevalencia del $40 \%$. Siendo ligeramente superior en varones $(44 \%)$ que en mujeres (42,8\%) según criterio OMS (Figura 1$)$.

Por culturas la prevalencia de malnutrición es algo superior en cristianos que en musulmanes, sin que dicha diferencia sea relevante. 
Figura 1

Gráfico en prisma acumulado de las malnutriciones según sexo y criterios de corte en los adolescentes de Ceuta

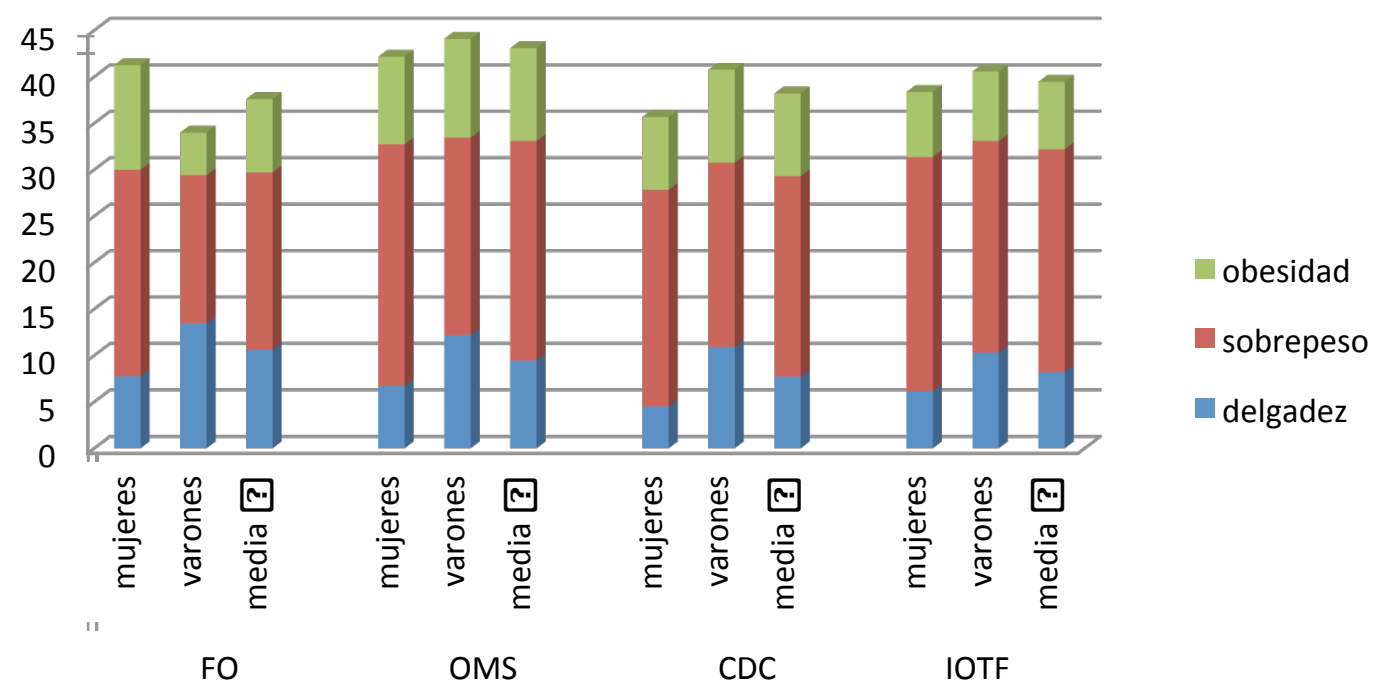

Cada día son más los investigadores ${ }^{9}$ que recomiendan realizar una acordada 0 concordancia de las prevalencias halladas con el PIMC con las encontradas con otras medidas, que ayuden a confirmar la situación nutricional individual y poblacional. Igualmente, desde el punto de vista de la salud, tanto del riesgo cardiovascular como del síndrome metabólico, resulta de gran importancia no solamente conocer la cantidad de grasa en el cuerpo sino cómo está distribuida dicha grasa en el organismo. Con estos fines, y mediante la medida del perímetro de cintura, se observa que la obesidad central o abdominal representa el $60 \%$ de los obesos, mientras que la obesidad generalizada lo es en el $40 \%$ de la población adolescente obesa.

Tomando como "referencia oro" el criterio de PIMC con puntos de corte OMS, se han calculado también las prevalencias de malnutrición según los perímetros de cadera, meso-braquial, y de muñeca. Las mejores correlaciones para nuestra población las encontramos entre el IMC, el perímetro de cintura y el perímetro meso-braquial (Tablas 3 y 4 ).

\begin{tabular}{|c|c|c|c|c|c|c|}
\hline \multicolumn{7}{|c|}{$\begin{array}{cc}\text { Tabla } 3 \\
\text { Prevalencia de malnutriciones por delgadez o d }\end{array}$} \\
\hline & IMC (OMS) & IBE & $\begin{array}{c}\text { Perímetro } \\
\text { Cintura } \\
\text { CCi }\end{array}$ & $\begin{array}{c}\text { Perímetro } \\
\text { Meso- } \\
\text { Braquial } \\
\text { PB }\end{array}$ & $\begin{array}{c}\text { Perímetro } \\
\text { Cadera } \\
\mathrm{CCa}\end{array}$ & $\begin{array}{c}\text { Perímetro } \\
\text { Muñeca } \\
\text { PM }\end{array}$ \\
\hline Varones Cristianos & 11,38 & 14,63 & 12,20 & & & \\
\hline $\begin{array}{l}\text { Varones Musulmanes } \\
\text { Total Varones }\end{array}$ & $\begin{array}{c}13 \\
12,9\end{array}$ & $\begin{array}{c}16,66 \\
15,8\end{array}$ & $\begin{array}{l}13,33 \\
12,87\end{array}$ & 9,90 & 12 & 11,88 \\
\hline Mujeres Cristianas & 4,25 & 5,55 & 5,55 & & & \\
\hline Mujeres Musulmanas & 9,20 & 10,25 & 10,25 & & & \\
\hline Total Mujeres & 6,72 & 8 & 8 & 7,33 & 6,66 & 13,33 \\
\hline $\begin{array}{l}\text { Total Muestra } \\
\text { IMC, IBE, CCi, PB, CCa, PM }\end{array}$ & 9,45 & 11,9 & 9,96 & 8,61 & 9,33 & 12,60 \\
\hline
\end{tabular}




\begin{tabular}{|c|c|c|c|c|c|c|}
\hline \multicolumn{7}{|c|}{$\begin{array}{c}\text { Tabla } 4 \\
\text { Prevalencia de malnutriciones por exc }\end{array}$} \\
\hline & IMC (OMS) & $\begin{array}{c}\text { IBE } \\
\text { EXCESO } \\
\text { DE GRASA }\end{array}$ & $\begin{array}{l}\text { Perímetro } \\
\text { Cintura } \\
\mathrm{CCi}\end{array}$ & $\begin{array}{c}\text { Perímetro } \\
\text { Meso- } \\
\text { Braquial } \\
\text { PB }\end{array}$ & $\begin{array}{c}\text { Perímetro } \\
\text { Cadera } \\
\mathrm{CCa}\end{array}$ & $\begin{array}{c}\text { Perímetro } \\
\text { Muñeca } \\
\text { PM }\end{array}$ \\
\hline Varones Cristianos & 34,28 & 31,7 & 34,14 & & & \\
\hline $\begin{array}{l}\text { Varones Musulmanes } \\
\text { Total varones }\end{array}$ & $\begin{array}{c}29,53 \\
31,9\end{array}$ & $\begin{array}{c}28,33 \\
29,7\end{array}$ & $\begin{array}{l}31,66 \\
32,67\end{array}$ & 31,7 & 30 & 23,72 \\
\hline Mujeres Cristianas & 38,73 & 33,3 & 36,11 & & & \\
\hline Mujeres Musulmanas & 32,10 & 29,5 & 33,33 & & & \\
\hline Total mujeres & 35,41 & 31,3 & 34,7 & 36 & 32,7 & 33,36 \\
\hline $\begin{array}{l}\text { Total Muestra } \\
\text { IMC IBE, CCi ,PB, CCa, PM }\end{array}$ & 33,65 & 30,5 & 33,86 & 33,85 & 31,35 & 28,54 \\
\hline
\end{tabular}

Un puntal importante a la hora de aumentar la sensibilidad para el diagnóstico y definición del infrapeso y del exceso de peso calculados a partir de las medidas antropométricas, es la Evaluación del Estado Nutricional sobre el contenido en grasa mediante la metodología de impedancia bioeléctrica tetrapolar ${ }^{12,9}$. Metodología clasificada como de fiabilidad media-alta. Los datos que nos reporta sobre la infra grasa son de un $15,84 \%$ en varones (cristianos $14,4 \%$, musulmanes $16,57 \%$ ) y de un $8 \%$ en mujeres (cristianas 6\%, musulmanas 10\%) (Figura 2).

Las edades con mayor prevalencia de malnutrición, tanto en mujeres como en varones adolescentes son de 13 a 15 años. Este método de impedancia infravalora la grasa corporal, sobre todo en los varones.

Figura 2

Porcentajes comparados de contenido en masa grasa por sexo y grupo etario

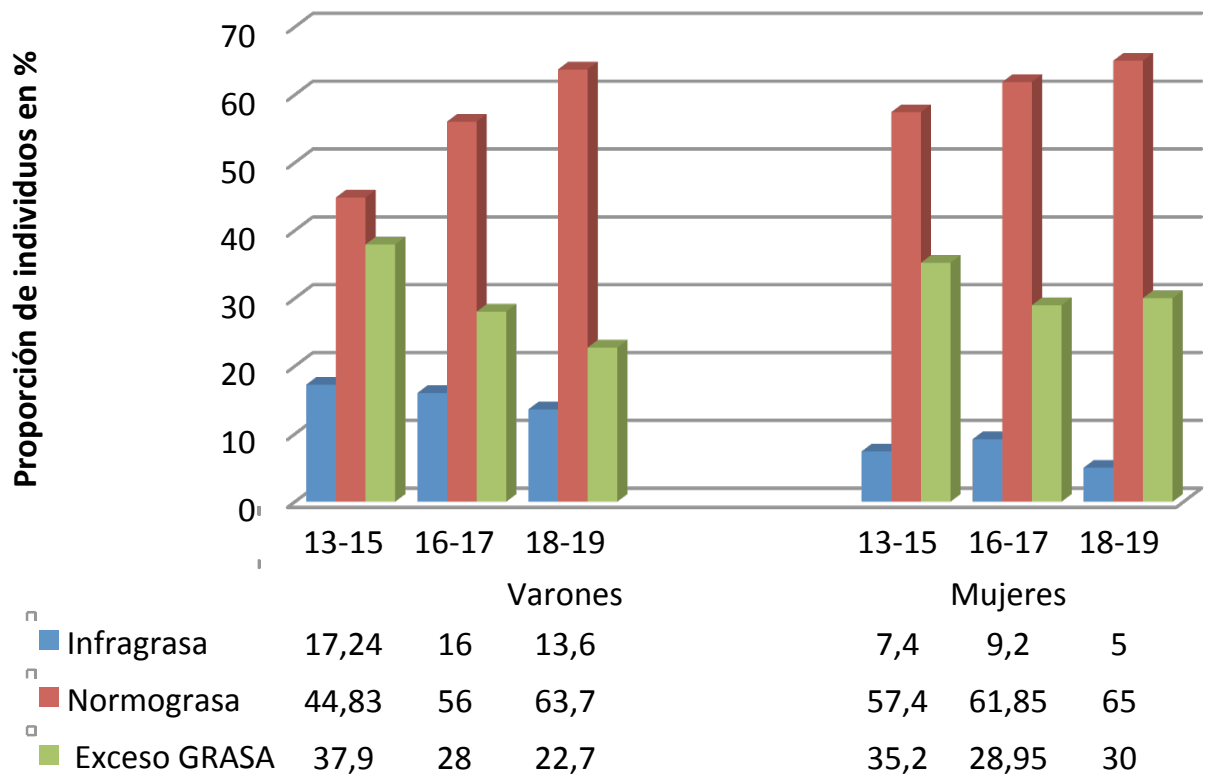

\section{Discusión}

El trabajo de investigación que discutimos es el primero que estudia con gran detalle el estado nutricional de los jóvenes adolescentes ceutíes con edades de 13 a 19 años, mujeres y varones, cristianos y musulmanes. Características de una población 
multicultural y pluriétnica. Por cristianos y musulmanes no nos referimos al hecho específico de practicar una religión. Desde las variables nutricionales y de composición corporal de esta investigación, nos interesan las características genéticas, antropológicas y sociológicas vinculadas a la generalidad de los que pertenecen a la cultura cristiana y a la cultura musulmana.

Hallamos las prevalencias de malnutrición con múltiples medidas directas antropométricas y de impedancia bioeléctrica, que aseguran una elevada fiabilidad. Además, el tamaño muestral, superior al $12,5 \%$ del total de la población adolescente, confiere a la investigación una elevada representatividad.

Por todo ello, se pretende de esta investigación -a la que damos el nombre de Estudio ABYLA- que sea un buen referente para futuras líneas de trabajo.

En relación a los objetivos planteados, establecemos las siguientes conclusiones:

- Sólo el $60 \%$ de los adolescentes ceutíes tiene un índice de masa corporal (IMC) dentro de los percentiles de normalidad. El 30\% presenta exceso de peso $(20 \%$ sobrepeso y $10 \%$ obesidad), y el $10 \%$ infrapeso o delgadez. Se observan pequeñas diferencias según sexo y cultura, siendo la obesidad ligeramente superior en musulmanes que en cristianos, y el sobrepeso mayor en cristianos que en musulmanes.

- La prevalencia de infrapeso o delgadez en la población joven de Ceuta es muy superior a la observada en otras regiones de España ${ }^{6}$. Ha aumentado sustancialmente en los últimos años hasta alcanzar un $10 \%$, correspondiendo un $3 \%$ a delgadez extrema.

- La prevalencia de sobrepeso hallada en nuestro estudio del 19,1\% (criterio FO) y del 23\%(criterio OMS) es superior al referido en estudios anteriores de universos más generales: Enkid -2000 (10\%), OMS-2010 (17\%), ENSE-2012 $(18 \%)$, ENSE-2014 (17\%). Se aprecia una tendencia hacia un aumento en la prevalencia del sobrepeso. Siendo esta situación, junto con la delgadez extrema, las que deben de afrontarse desde todos los ámbitos: educativo, de salud comunitaria, y de política social. Las expectativitas y su calidad de vida de nuestra población adolescente van en ello.

- La prevalencia de obesidad es la población de jóvenes de Ceuta (10\% -OMS y $8 \%-F O)$ es inferior a la referida en el Estudio Enkid ${ }^{14}$ para la media nacional $\left(13,5 \%\right.$ FO). Pudiéndose confirmar que la prevalencia de obesidad ${ }^{10,13}$ ha disminuido en general en España en la última década, y en Ceuta en particular. Ocupando Ceuta un lugar por debajo de la media nacional.

- Los diversos criterios para la definición de las prevalencias de malnutrición ofrecen valores muy semejantes (OMS, FO, IOTF, CDC). Siendo los puntos de corte FO más sensibles para la delgadez y menos sensibles para el exceso de peso en varones.

- Existe, en general, una buena concordancia entre el exceso de peso y el infrapeso o delgadez definidos por el PIMC y lo que se determina por los percentiles de diámetro de cintura, diámetro del brazo-medio, y por la impedancia bioeléctrica. 
- Al pasar de la niñez a la adolescencia se produce una disminución significativa de la prevalencia de obesidad, que pasa del 25,3\% descrito en el Estudio Aladino 2011 (criterio PIMC-OMS) para niños y niñas de 6 a 9,9 años de Ceuta , a un $10 \%$ en los adolescentes de 13 a 19 años hallado en nuestro Estudio Abyla.

- Es el grupo etario de 13-15 años el que presenta (tanto en varones como en mujeres, en cristianos como en musulmanes) mayores niveles de exceso de grasa.

- No se aprecian diferencias significativas en las prevalencias de malnutriciones alimentarias en razón a la etnia o genética. Más bien parecen ser la moda y las costumbres alimentarias del entorno social del adolescente los factores que determinan el estado nutricional en este lugar, crisol de culturas, que llamamos Ceuta.

\section{Referencias}

1. Agencia Española de Seguridad Alimentaria (2005). Estrategia NAOS. Madrid: Ministerio de Sanidad y Consumo.

2. Aranceta-Bartrina, J., Pérez-Rodrigo, C., Ribas-Barba, L. y Serra-Majem, L. (2005). Epidemiología y Factores determinantes de la obesidad infantil y juvenil en España. Revista Pediátrica de Atención Primaria, 7(1),13-20.

3. Briz-Hidalgo, FJ., Cos-Blanco, I. y Amate-Garrido, AM. (2007). Prevalencia de obesidad infantil en Ceuta. Estudio PONCE 2005. Nutrición Hospitalaria, 22(4),471-7.

4. Cobo, P., Santana, A., Berrocal, E., Dúo, P.(2006). Descripción del patrón de consumo de golosinas en escolares de $5^{\circ}$ curso de Educación Primaria de la Ciudad Autónoma de Ceuta. Revista Española de Nutrición Comunitaria,12(1),6-13.

5. Estudio ALADINO. (2013). Estudio de Vigilancia del crecimiento, Alimentación, Actividad física, Desarrollo infantil y Obesidad en España 2011. Madrid: Agencia española de Seguridad Alimentaria y Nutrición.

6. García-Fuentes, M. (2007). Obesidad infantil en España. Epidemiología, comorbilidades y prevención, con especial referencia a la población adolescente. Madrid: Sociedad de Pediatría Social.

7. Gil-Hernández, A. (2010). Tratado de Nutrición: valoración del estado nutricional. Madrid: Ángel Gil Hernández y SENPE.

8. Hernández, M., Castellet, J. y Narvaiza, JL. (1988). Curvas y tablas de crecimiento. Bilbao: Fundación Orbegozo.

9. Martin-Moreno, V., Gómez-Gandoy, JB. y Antoranz-González, MJ. (2001). Mediciones de la grasa corporal mediante impedancia bioeléctrica, pliegues cutáneos y ecuaciones a partir de medidas antropométricas. Análisis comparativo. Revista Española de Salud Pública, 73, 221-236.

10. Martínez-Álvarez, JR., Villarino-Marin, A., García-Alcón, RM., Calle-Purón, ME. y Marrodan-Serrano, MD. (2013). Obesidad infantil en España: hasta qué punto es un problema de salud pública o sobre la fiabilidad de las encuestas. Nutrición Clínica y dietética hospitalaria, 33 (2) ,80-88.

11. Martínez-Gómez, D., Eisenmann, JC., Gómez-Martínez, S., Veses, A. y Marcos, A. (2010). Sedentarismo, adiposidad y factores de riesgo cardiovascular en adolescentes. Revista Española de Cardiología, 63, 27785.

12. Martínez-Sopena, MJ., Redondo-Del Río, MP. y Alonso-Franch, M. (2006). Valoración del estado nutricional del obeso: estimación de la masa grasa. Boletín de la Sociedad de Pediatría de Asturias, Cantabria, Castilla y León.46, 275-291.

13. Sánchez-Cruz, JJ., Jiménez-Moleón, JJ., Fernández-Quesada, F. y Sánchez,M.J. (2013).Prevalencia de obesidad infantil y juvenil en España en 2012. Revista Española de Cardiología, 66, 371-6.

14. Serra-Majem, L., Ribas-Barba, L. y Aranceta-Bartrina, J. (2003). Obesidad infantil y juvenil en España. Resultados del Estudio Enkid 1999-2000. Med. Clin,121(19), 725-32.

15. Taylor, RW., Jones, JE., Willians, SM., \& Goulding, A. (2000). Evaluation of waist circumference, waist-tohip, and the conicity index as screening tools for high trunk fat mass, as measured by dual-energy X-ray absorptiometry, in children aged 3-19y. American Journal of Clinical Nutrition, 72, 490-5. 